\title{
A New Integrated Front Platform of Financial Self-service Equipment Based on ESB
}

\author{
Juan Xiao \\ Institute of Electrical and Electronic \\ Engineering, \\ Wuhan Polytechnic University , \\ Wuhan,430023, China \\ e-mail:susanxjuan@163.com
}

\author{
Juan Wu \\ R\&D Center, \\ Wuhan Tianyu Information Industry \\ Co., Ltd. \\ Wuhan,430223, China \\ e-mail:janice79@126.com
}

\author{
Jiagong Yang* \\ No.3 Department, \\ Air Force Radar Academy, \\ Wuhan, 430012,China \\ e-mail: yxtyt0310@163.com \\ *corresponding author: Jiagong Yang
}

\begin{abstract}
Enterprise Service Bus (ESB) is a kind of SOAbased software infrastructure for the enterprise application integration. ESB becomes a hot technology of channels and resources integration.In this paper, in view of the characteristics of self-service channels and its IT architecture, we propose the scheme design of a new ESB-based integrated front platform of financial self-service equipments.It is called ProTeller ESB. The platform combines the functions of the self-service channels and front system to achieve a standardized service.It makes the IT structure of self-serive with good robustness and scalability, and can construct the ESB system fastly and efficiently.It can enhance the capacity of the existing system restructuring.
\end{abstract} ESB

Keywords- SOA;self-service equipment;channel Introduction;

\section{INTRODUCTION}

With the unceasing development of the domestic bank services and IT technology changing, it has caused many kinds of bank internal systems and the application types. IT schema of bank is also very complex. The relationship between the bank and the customer has also had the huge change. Faced with the changing and challenge, the bank needs urgently to integrate the channels and resources, facilitate the rapid development the middle business and can adapt the rapidly the market changing.

The channel is the carrier of the banking market's sales, and is the competitive power importantly manifests in nowaday bank. At present the bank channel way mainly has three kinds,that is the counter channel, self-service channel and electronic channel. The research indicated that the channel conveniences are the primary factor for the consumer to choose the banking service. Therefore, the channel plays the decisive role for the market sales of the retail bank. Faced with the multiplex tendency of the retail channels in banking system, IT schema and the platform of the current bank already could not conform to business demand of the development and the challenge which multichannel brought. The banking industry is facing the IT schema reforming bottleneck. The multi-channel integration is the solution of current bottleneck from the front channel, the middle business platform to all kinds of banking systems.
It is best selection which conforms to the demand of the bank business development.

The self-service channel, which is provided the selfserivce for customers by the automatical deposit/withdraw machines, self-service terminal and POS in the specific place by the bank or the channel partner, is the most important supplement to the counter channel in the banking industry. It is one of main channel which the bank will future develop.

Therefore, this article proposed a new ESB-based integrated front platform of financial self-service equipments with multi-level, the standardization and the loosely coupling, that is, ProTeller ESB. ESB plays the visit pivotal role for the visit between the services int the IT's schema. It is the foundation and the breakthrough point to build SOA framework.

\section{SOA AND ESB}

\section{A. Service-Oriented Architecture SOA}

Service-oriented architecture (Service Oriented Architecture, SOA) is one kind of software architecture model of an integrated service-based definition. SOA realizes the business process by the service's call and the combination. Service is a discrete functional unit, it is packaged the reusable business logic. Its character is the platform independence, location transparency and interoperability. It also provides the standard interface of the external calling. According to the demand, the losely coupled sevices can be distributed to deploy, be combined and be used. The goal of SOA is to enable the IT system becomes more flexible and more rapid to response the changing business needs[1].

WEB-based SOA technology may achieve its model by XML, SOAP, WSDL, UDDI and other technologies. The content and the form of the request message and reply message can be described 8by the XML technology. The services can be descibed by the WSDL technology. The message format of the service provider and service requestor can be described by the SOAP technology. The registrated format of the standard services can be described by the UDDI technology. 


\section{B. Enterprise Service Bus(ESB)}

The Enterprise Service Bus (ESB) is the infrastructure which underpins a fully integrated and flexible end-to-end service-oriented architecture(SOA). The ESB definition is : "The ESB is an open standards-based technology concept that will revolutionize IT and enable flexible and scalable distributed computing for generations to come. The ESB is emerging as the backbone of the distributed framework within enterprise IT, because it allows not only the retention and deployment of existing business-critical applications, but also allows the user to introduce and remove newer applications as needed." [3] [4] The ESB architecture is shown in figure 1.

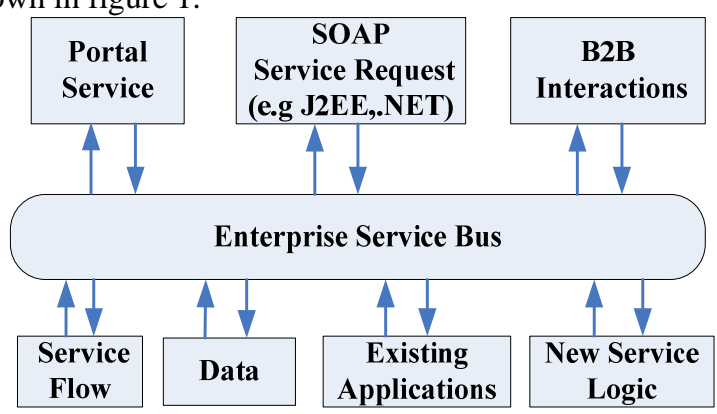

Figure 1.Enterprise Service Bus

ESB is a loosely coupled, distributed, event-driven enterprise-class SOA. ESB is a tchnology integration of traditional middleware technology, XML, Web services and so on technology. For enterprises, you may not need to add any software and hardware devices, may integrate the past, existing and the future IT system to the enterprise-level application framework by using the ESB middleware system. ESB can provide real-time, the large capacity information correspondence and the capabilities of the realtime control, the management and assignment news transmission.

ESB has overcome the flaw of the traditional EAI technology, can provides the support to each kind of technology and the application system. ESB has the very strong flexibility and the extendibility.The ESB model is helpful to reduced application connection quantity and the complexity, thus can reduce the IT maintenance and the change cost. It lets the enterprise be able to use the service by simpler, faster, a safer way[5].

\section{ESB-BAsed SOlution OF FinANCIAL SELF-SERVICE EQUIPMENT}

\section{A. The present situation of self-service channels}

Along with IT technology development and new service unceasing change, the IT framework of the current bank exists a very big bottleneck, it has restricted the bank service development. It Mainly manifests in the following several aspects:

1) Complex IT foundation construction day by day:
- Bank systems of the core business system, credit card system and all kinds of middleware business are established by the dispersal and the independent way.

- The diversification of message exchange standards and communication protocols.

- Widespread application of middleware, heterogeneous platforms and databases.

2) Complex business model day by day:

- Between the service process is alternately more complex and the service process differentiation.

- The technical development actuates the new cooperation model[2].

As a result of existing many drawbacks of dispersed business process logic, the tight coupling way between the systems and lack of a unified integration of norms, the baning system becomes very difficult to miantain and expand, although bank used the big front systems to integrate resources.

In IT schema of the self-service service channel, because the self-service front system must be connected with the multitudinous banking front systems with the sole function, its periphery connection becomes to be more and more many, its management becomes to be more and more complex. Simultaneously, once the operational channel changes, the development and the software updating will become to be diffcult for the self-service channels. In addition, because there are many kinds of self-service devices, it also increased the difficulty to develop and update the applications, business is not conducive to rapid promotion. The applications will lack the flexibility and the extendibility[6] [7].

\section{B. Architecture}

In order to solve the problem which the current selfservice channel exists, and to adapt the middle-service unceasing change and development, we must completely overcome the drawbacks, such as the single function of the self-service front system, the ability to focus on forward transactions, lacks of the system integration function and the flow management function, and so on. This article combines the advanced international banks on the leading ideas of the Enterprise Service Bus and best Practices, we propose the scheme design of a new ESB-based integrated front platform of financial self-service equipments, that is ProTeller ESB.This platform architecture is shown in Figure 2.

ProTeller ESB is a fundation platform of SOA-oriented ESB, also is the WEB-based standard services. It overcomes the drawbacks of the traditional EAI technology. ProTeller ESB is an open-standard message-based middleware that provides routing, invocation, and mediation services to facilitate the interaction of disparate distributed infromation technology resources in a reliable manner. 


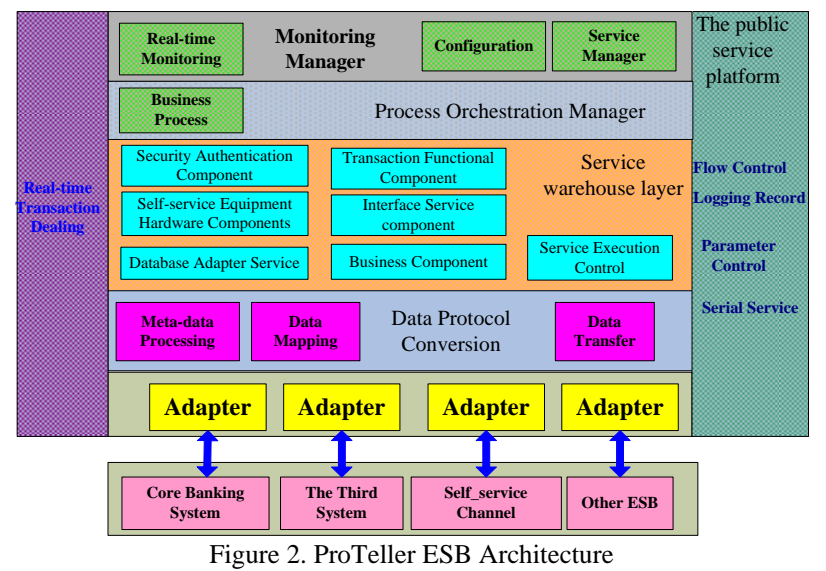

\section{Functional descriptions}

The primary function of ProTeller ESB are as follows:

1) Adapters layer: The adapter layer provides a unified platform for access to capacity for the self-service, the backstage application system, and the third party system by adapters. Simultaneously it provides to the asynchronous processing support.

2) Data protocol conversion: To provide a unified protocol conversion of the message format, communication protocols, as well as the norms of financial services. It provides the tools with meta-data processing, data mapping, data translation functions. It realizes the standardization of data modeling and data format conversion and mapping, and provides the the convenience for the data integration between the application systems.

3) Service warehouse layer: According to the norms of the standard service and the XML technology, the service components that the original system and new business system components required will be packaged. It constitutes a service container, that is, the storage layer service component. The main service components are as follows:

- Security Authentication Component: By the key management, authentication, communication encryption, and MAC of the essential field, it has established a complete set of security mechanisms to ensure the safe operation of the entire system.

- Self-service Equipment Hardware Components: The component has realized the service interfaces and the service process for each functional module of the financial self-service devices by the CEN/XFS standard norms. The mainly hardware equipments include deposit,withdraw,printer,keypad,IC card Reader,handheld terminal and etc.

- Transaction Functional Component: It has realized the function components which the self-service equipment mainly completes, such as withdrawals, deposits, modify password, query, query the transaction details, transfer, IC card transactions (optional), payment services (optional ), as well as self-service equipment maintenance function modules.

- $\quad$ Interface Service component: It has mainly realized the display about the advertising and transaction screen interface of the self-service equipment.

- Business Component: It has provided the banking business components for integrated resources and middle business, and has packaged to atom banking services.

- Database Adapter Service: It has the packaged servcie and event-driven features. It has provided the data extraction, packing, storage and other function by the user configuration. It supports current mainstream databases, such as Oracle, DB2, Infromix, Mysql,and etc.

- Service Execution Control: It has provided the verification, the control and the switch for the executing service state, simultaneously it has realized the service dynamic discovery and the message routing mechanism.

4) Process Orchestration Manager: The adapter layer provides a unified platform for access to capacity for the self-service, the backstage application system, and the third party system by adapters. Simultaneously it provides to the asynchronous processing support.

5) Monitoring management layer: Statistics for key message and transport attributes. Message logging with archive and search. Enables detailed, real-time monitoring, dynamic resource loading and service configuration.

6) The real-time transaction transfer processes the platform: It provides fast processing channel to real-time transaction. It mainly provides the transfer function of the real-time transaction to the bank core business system. This module needs to provide the highly effective performance and safe processing, simultaneously records the local diary to guarantee the transaction integrity.

7) The public service platform: The public service includes the unification the flow control service, the serial number service, the asynchronous running record, the logging record, and etc. It realizespublic service support of the multi-technical platform and multi-physical deployment environment[9] [10].

\section{CONCLUSIONS}

The ProTeller ESB is a ESB-based development platform of self-service device. It has achieved the unified functions of the routing, service definition, distribution and management. It also supports flexible business models. ProTeller ESB may fully adapt to the process changing about the self-service equipment, banking background applications and the middle business.It provides a new platform in business innovation. Its monitoring platform realizes the unity of monitoring management. More importantly, the entire system uses the unified service development framework, achieves a unified standard development ways, reduces overall system development and 
maintenance cost and improves the reorganized capacity of the existing system. It can help banks to establish a rapid and efficient ESB system [8].

The multilevel nature of ProTeller ESB, standardization, the strip lines and loosely coupling, enables the ESB platform to have the high performance, the high usability and expansion ability. ProTeller ESB realizes seamless integration with the third party ESB product, can adapt fully the IT environment of bank.

\section{REFERENCES}

[1] Lv Hongwei. The WEB Service technology of SOA architecture [J] Computer Programming Skills \& Maintenance, 2010 (4)

[2] S.Vinoski, "Integration with Web Services", IEEE Internet Computing, vol. 7, no. 6, IEEE, 2003, pp. 75-77.

[3] D.A. Chappell, “Enterprise Service Bus”, O’Reilly Publishing, 2004
[4] M.T. Schmidt, B. Hutchison, and P. Lambros, "The nterprise Service Bus: Making Service-Oriented Architecture real”, IBM Systems Journal, vol.44, no.4, 2005, pp. $781-797$

[5] Rajam,S.; Cortez,R.; Vazhenin,A.; Bhalla,S. "Enterprise Service Bus dependency injection on MVC design patterns" [C] TENCON 2010,2010 IEEE Region 10 Conference,2010:PP1015-1020

[6] Xu Ruzhi; Bai Jin; Wang Yufei, "The research and implementation of power application system integration based on enterprise service bus" [C] Intelligent Computing and Intelligent Systems (ICIS), 2010:PP 466-469

[7] Jihong Yan; Wenrong Jiang; Shiliang Zheng; Cuihong Wu; Hailan Pan, "Research on application of Web based ESB in School Common Data Platform ” Computer Science \& Education, 2009. ICCSE '09. Int. Conf. PP: $1311-1315$

[8] Jr. Ortiz, Sixto, "Getting on Board the Enterprise Service Bus", Computer, vol.40, no.4, 2007, pp.15 - 17

[9] http://www.searchsoa.com.cn/index.htm

[10] http://www.ibm.com/developerworks/cn/webservices/ 